\title{
Mouse models of visual deficits
}

\section{To the editor:}

In their recent article ${ }^{1}$, Ambati and colleagues present important data that provides insight into cell involvement in age-related macular degeneration (AMD). However, they erroneously state that there is "no animal model of drusen deposition or spontaneously occurring $\mathrm{CNV}$ [choroidal neovascularization] resembling that seen in patients with AMD." Jackson Laboratories (Bar Harbor, Maine, USA) has a strain of mouse $(\mathrm{C} 57 \mathrm{BLKS}-\mathrm{Bst} / \mathrm{J})$ in which, "after seven months of age, $19 \%$ of heterozygotes have focal, nonrhegmatogenous, retinal detachment accompanied by subretinal neovascularization." These symptoms of this strain were originally reported by Smith et al. ${ }^{2}$. Although Ambati et al. present an exciting finding of a new model for AMD, it would benefit the readers to be aware that there is at least another model of CNV that may also suit their research interests.

\section{Nilanjana Sengupta \& Sergio Caballero}

Department of Pharmacology and Therapeutics, PO Box 100267, University of Florida, Gainesville, Florida 32610-0267, USA. jhimli@ufl.edu

1. Ambati, J. et al. Nat. Med. 9, 1390-1397 (2003). 2. Smith, R.S. et al. Proc. Natl. Acad. Sci. USA 97 2191-2195 (2000).

\section{Ambati et al. reply:}

We thank Sengupta and Caballero for their interest in our work. Although a small frac- tion of $\mathrm{Bst}^{+/-}$mice spontaneously develop choroidal neovascularization (CNV), Sengupta and Caballero do not mention that a majority of these heterozygous mice show many other severe ocular disturbances $^{1-3}$. Nearly $60 \%$ of $\mathrm{Bst}^{+/-}$ mice have abnormal pupillary light responses owing to unilateral or bilateral atrophy of the optic nerves. The mean number of retinal ganglion cells is reduced by $70 \%$ as a result of delayed fusion of the optic fissure. In addition to optic nerve malformation (coloboma), several foci of the neural retina are characterized by folds, dysplasia and thinning. Ectopic ganglion cell axons and photoreceptor nuclei are further examples of disorganization. Persistence of the hyaloid vessels is another ocular disturbance in $B s t^{+/-}$mice.

Although the optic nerve pathology in $B s t^{+/-}$mice is vaguely reminiscent of autosomal dominant optic atrophy (DOA) in humans, it also includes numerous other ocular abnormalities not resembling those seen in DOA. In addition, the mouse ortholog of the human OPA1 gene, which is mutated in DOA, has been excluded from the mouse Bst locus ${ }^{4}$. Given that the CNV phenotype in $\mathrm{Bst}^{+/-}$mice is even more distant than the optic nerve abnormality from the respective human conditions, CNV in these mice is likely to be as uninstructive about the pathogenesis of the human condition as, say, the appearance of lens opaci- ties in these mice is to be about age-related cataract in patients. The ocular phenotype of patients with early or late AMD bears no resemblance to the panoply of structural abnormalities in $\mathrm{Bst}^{+/-}$mice, which probably result from global embryonic dysfunction rather than specific perturbations of cellular pathways. Nevertheless, given the rare occurrence of spontaneous $\mathrm{CNV}$ in mice, study of this angiogenic process will provide insight into the secondary effects of $\mathrm{CNV}$ on photoreceptor disruption, which ultimately leads to deficits in visual perception.

Jayakrishna Ambati ${ }^{1}$, Akshay Anand ${ }^{2}$, Eiji Sakurai $^{1}$, William A Kuziel ${ }^{3}$, Barrett J Rollins ${ }^{4}$ \& Balamurali K Ambati ${ }^{5}$

${ }^{1}$ Department of Ophthalmology \& Visual Sciences, University of Kentucky, Lexington, Kentucky 40536-0284, USA. ${ }^{2}$ Department of Neurology, Postgraduate Institute of Medical Education and Research, Chandigarh, 160012 India. ${ }^{3}$ Protein Design Labs, 34801 Campus Drive, Fremont, California 94555, USA.

${ }^{4}$ Dana Farber Cancer Institute, Harvard Medical School, Boston, Massachusetts 02115, USA.

${ }^{5}$ Department of Ophthalmology, Medical College of Georgia, Augusta, Georgia 30907, USA.

e-mail:jamba2@uky.edu

1. Rice, D.S. et al. Invest. Ophthalmol. Vis. Sci. 38, 2112-2124 (1997).

2. Tang, Q., Rice, D.S. \& Goldowitz, D. Dev. Biol. 207, 239-255 (1999).

3. Smith, R.S. et al. Proc. Natl. Acad. Sci. USA 97, 2191-2195 (2000)

4. Delettre, C., Lenaers, G., Belenguer, P. \& Hamel, C.P. BMC Genet 4, 8 (2003). 